\title{
Effective number of white shark (Carcharodon carcharias, Linneaus) breeders is stable over four successive years in the population adjacent to eastern Australia and New Zealand
}

\author{
Danielle Davenport ${ }^{1}$, Paul Butcher ${ }^{2}$, Sara Andreotti ${ }^{3}$, Conrad A. Matthee ${ }^{3}$, Andrew \\ Jones $^{4}$, and Jennifer Ovenden ${ }^{1}$ \\ ${ }^{1}$ The University of Queensland \\ ${ }^{2}$ NSW Government \\ ${ }^{3}$ Stellenbosch University \\ ${ }^{4}$ The University of Queensland School of Biomedical Sciences
}

September 24, 2020

\begin{abstract}
Population size is a central parameter for conservation, however monitoring abundance is often problematic for threatened marine species. Despite substantial investment in research, many marine species remain data-poor resulting in uncertain population forecasts and restricting the evaluation of past and present conservation actions. Such is the case for the white shark (Carcharodon carcharias), a highly mobile apex predator for whom population monitoring is a conservation priority following substantial declines recorded through the 20th century. Here, we estimate the effective number of breeders that successfully contribute offspring in one reproductive cycle $(\mathrm{Nb})$ providing a snapshot of recent reproductive effort in an east-Australian New Zealand population of white shark. Nb was estimated over four consecutive age cohorts (2010, 2011, 2012, 2013) using two genetic estimators (linkage-disequilibrium; LD and sibship assignment; SA) based on genetic data derived from two types of genetic markers (single-nucleotide-polymorphisms; SNPs and microsatellite loci). While estimates of Nb using different marker types produced comparable estimates, microsatellite loci were the least precise. The LD and SA estimates of Nb within cohorts using SNPs were comparable, for example the 2013 age-cohort Nb(SA) was 289 (95\%CI 200-461) and Nb(LD) was 208.5 (95\% CI 116.4-712.7). We show that over the time period studied Nb was stable and ranged between 206.1( \pm 45.9$)$ and $252.0( \pm 46.7)$ per year using a combined estimate of $\mathrm{Nb}(\mathrm{SA}+\mathrm{LD})$ from SNP loci, and a simulation approach showed that in this population effective population size $(\mathrm{Ne})$ per generation can be expected to be larger than Nb per reproductive cycle. This study demonstrates how breeding population size can be monitored over time to provide insight into the effectiveness of recovery and conservation measures for the white shark, where the methods described here may be applicable to other data-poor species of conservation concern.
\end{abstract}

\section{Hosted file}

Davenport_etal_White_Shark_MS_Revised.pdf available at https://authorea.com/users/361548/ articles/482915-effective-number-of-white-shark-carcharodon-carcharias-linneausbreeders-is-stable-over-four-successive-years-in-the-population-adjacent-to-easternaustralia-and-new-zealand 